\title{
Thermal and Mechanical Performance of the First MICE Coupling Coil and the Fermilab Solenoid Test Facility
}

\author{
Roger Rabehl, Ruben Carcagno, Shlomo Caspi, Allan DeMello, Lidija Kokoska, Darryl Orris, Heng Pan, Cosmore \\ Sylvester, and Michael Tartaglia
}

\begin{abstract}
The first coupling coil for the Muon Ionization Cooling Experiment (MICE) has been tested in a conductioncooled environment at the Solenoid Test Facility at Fermilab. An overview of the thermal and mechanical performance of the magnet and the test stand during cool-down and power testing of the magnet is presented.
\end{abstract}

Index Terms-Cryogenics, Strain measurement, Thermal analysis, Test facilities.

\section{INTRODUCTION}

$\mathrm{A}$ NEW TEST facility has been constructed at the Fermilab Central Helium Liquefier (CHL). The Solenoid Test Facility (STF) provides a unique capability for high-power testing of conductively-cooled superconducting solenoid magnets under cryogenic conditions. Siting STF away from other Fermilab test facilities allows large fringe magnetic fields to be safely accommodated without impacting personnel or equipment. The cryogenic requirements are handled by the CHL plant, which provides a dedicated liquid helium supply so that other test programs are not affected.

The first test subject was a coupling coil solenoid for the Muon Ionization Cooling Experiment (MICE) to be operated at Rutherford Appleton Laboratory in the United Kingdom. The solenoid was wound with 96 layers, 166 turns per layer, of copper-stabilized niobium-titanium superconductor. At $210 \mathrm{~A}$, the magnetic flux density at the center of the coil is $2.6 \mathrm{~T}$, and the peak magnetic flux density in the coil is $7.5 \mathrm{~T}$. Other details of the coil and the magnet construction can be found elsewhere [1]-[4].

The test stand design and features, facility subsystems, and initial operating experience are described elsewhere [5]. After overcoming many obstacles, the magnet reached a quench current of 195 A. This was below the 214 A target current but above the 175 A minimum operating current for MICE. The

Fermi National Accelerator Laboratory is operated by Fermi Research Alliance, LLC under Contract No. DE-AC02-07CH11359 with the United States Department of Energy. Lawrence Berkeley National Laboratory is operated under Contract No. DE-AC02-05CH11231 with the United States Department of Energy.

R. Rabehl, R. Carcagno, L. Kokoska, D. Orris, C. Sylvester, and M. Tartaglia are with Fermi National Accelerator Laboratory, Batavia, IL 60510 USA, e-mail: rabehl@fnal.gov.

S. Caspi, A. DeMello, and H. Pan are with Lawrence Berkeley National Laboratory, Berkeley, CA 94720 USA. thermal performance and the mechanical performance of the magnet and the test stand during this ultimately successful test are presented here.

\section{THERMAL PERFORMANCE}

The first cool-down started on May 6, 2013. A cold helium leak appeared once two-phase helium reached the magnet outlet, degrading the insulating vacuum level to $2 \mathrm{~Pa}$ (15 mTorr). The conductively-cooled power leads, designed with the upper section optimized for 300-60 K operation and the lower section optimized for $60-4.5 \mathrm{~K}$ operation, were insufficiently cooled. With no current the intercepts reached only $85 \mathrm{~K}$ and $20 \mathrm{~K}$. The cold mass never become fully superconducting, with cold mass surface temperatures reaching only 8-11 K. As a result of subsequent warm leakchecking, a leak was found at a metal-to-metal face seal fitting. A flexible hose was installed to reduce stress on this joint, and the connection was remade. For the current leads, a more detailed thermal analysis was required. The analysis was updated, and a redesigned thermal intercept system was fabricated and installed. A subsequent zero-magnet test, with the cryostat piping modified to bypass the magnet and the current leads shorted, proved that the cryostat piping now remained leak-tight at cryogenic conditions, and the current leads were successfully ramped to 210 A although additional modifications to the thermal intercepts were required to stabilize the temperatures.

The cryostat was warmed up and the magnet was reconnected to the current leads. In this second cold run, cold mass surface temperatures improved relative to the initial cool-down but still reached only 5.2-7.5 K. The cold mass warm-up rate upon stopping the liquid helium supply flow indicated a heat load of $70-75 \mathrm{~W}$, well above the $10 \mathrm{~W}$ expected heat load and the $15 \mathrm{~W}$ maximum allowable heat load to keep the coil superconducting when at full power. The problem was identified as improper installation of the multilayer insulation (MLI). All seams had been taped over, preventing good vacuum from being established in the MLI blankets. The MLI was wrapped too tightly, and it was installed as two 20 layer blankets resulting in thermal shorts. Finally, no MLI was installed on the vertical support rods that support the weight of the cold mass from the cryostat top plate.

A significant effort to address the excessive heat load was 
then undertaken. Since the cryostat does not have a thermal shield, a custom thermal shield was designed and installed around the cold mass and the mechanical supports. The cold mass was first wrapped in a layer of $7.6 \times 10^{-5} \mathrm{~m}(0.003 \mathrm{in})$ thick alloy 1235, 99.35\% aluminum foil. A stainless steel cooling pipe was added above the cold mass. The helium gas return from the magnet flowed through this pipe which served as the thermal sink for thermal intercepts. As part of the thermal shield design, stainless steel tabs were welded to the cooling tube along its length. Fig. 1 shows the foil-wrapped cold mass with the cooling pipe above it. Fig. 2 is a close-up of the cooling pipe and its welded tabs. Flexible straps comprising part of the mechanical support thermal intercept system can also be seen in Fig. 2.

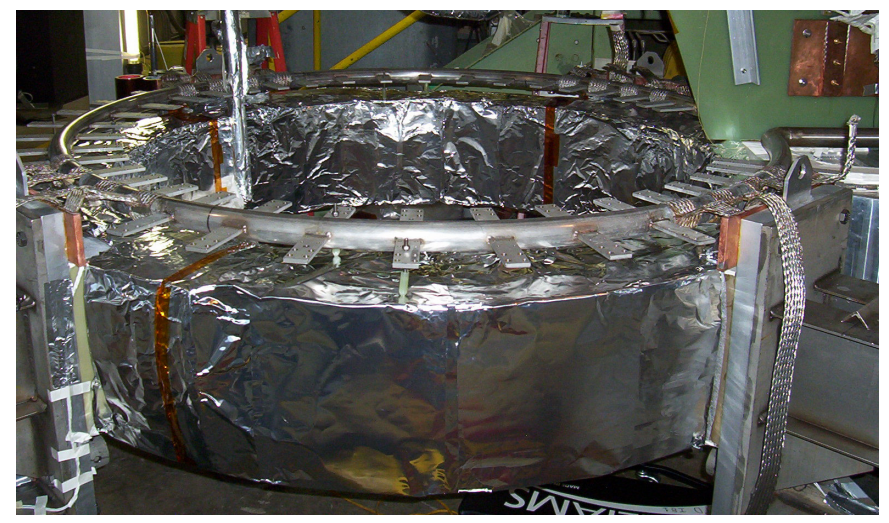

Fig. 1. The aluminum foil-wrapped cold mass with the added cooling pipe.

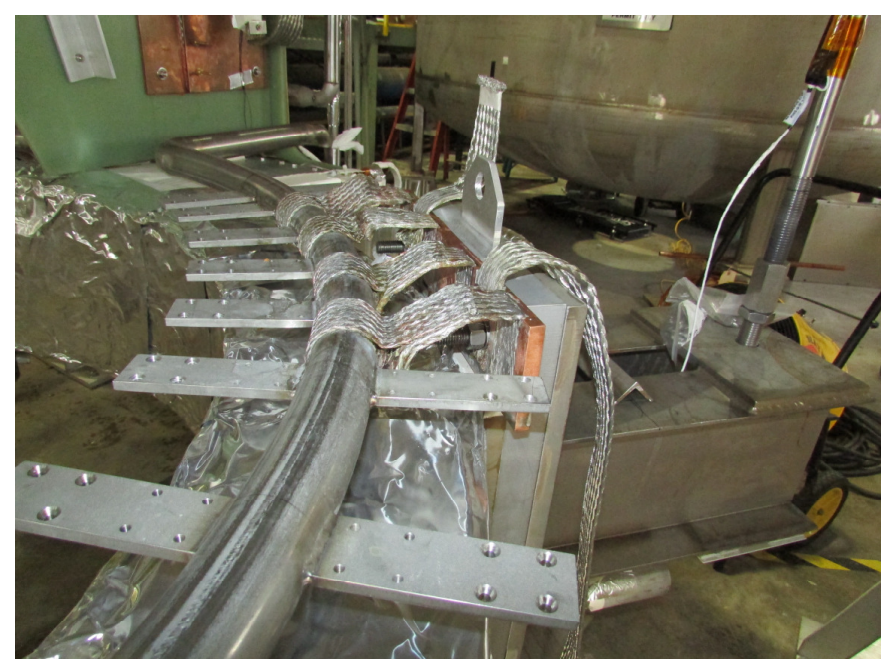

Fig. 2. The cooling pipe with welded tabs. One of the magnet supports can be seen to the right, and thermal intercept flexible straps can be seen at the center.

Aluminum shield boxes were built around the mechanical supports and thermally sunk to the cooling pipe. Aluminum ribs were bolted to the cooling pipe tabs, forming a cage around the cold mass. Fig. 3 is a 3-D model showing a crosssection of the cold mass, the cooling pipe, the aluminum rib cage, and an aluminum shield box around a mechanical support. A vertical support rod and a lateral support pin that prevents lateral motion of the cold mass during power testing are also shown in Fig. 3.

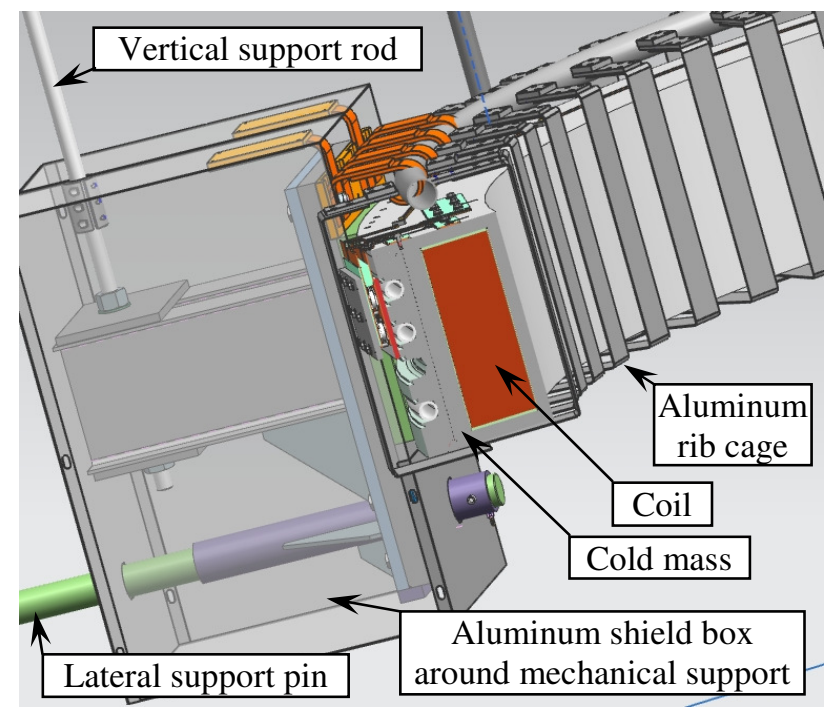

Fig. 3. A 3-D model of the magnet coil and the custom thermal shield components. A vertical support rod and a lateral support pin can be seen to the left.

A layer of aluminum foil was then wrapped over the aluminum rib cage and thermally sunk by clamping it to the stainless steel welded tabs. Use of Apiezon- $\mathrm{N}$ grease on these and all other mechanically clamped joints ensured good thermal contact.

The entire assembly was wrapped in 40 layers of MLI, consisting of alternating layers of double-aluminized mylar and Reemay. The MLI was applied in 5-layer blankets. Slits, $0.051 \mathrm{~m}$ ( 2 in) long and spaced $0.15 \mathrm{~m}$ (6 in) apart, were made in all blankets to improve the interlayer vacuum pump-out. Careful attention was paid to alternating wrapping directions, offsetting seams, minimizing taping, transitioning from warm areas to cold areas, and loose wrapping of the MLI.

In addition to installing a custom thermal shield and reinsulating the assembly, additional steps were taken to improve operations and troubleshooting capabilities. The insulating vacuum system was modified to bring the $400 \mathrm{l} / \mathrm{s}$ turbomolecular high-vacuum pump as close to the cryostat as possible, with the caveat that it would be isolated and turned off prior to magnet powering due to possible detrimental effects of the high fringe magnet field. A residual gas analyzer, a cold cathode sensor, and a multi-sensor vacuum transducer were added to provide verification that the helium system remained leak-tight and to provide continuous monitoring of the vacuum level. A vent pipe was installed on the aluminum rib cage surrounding the cold mass, allowing the vacuum space between the cold mass and the MLI blankets to be pumped out in common with the cryostat insulating vacuum space. Finally, temperature sensors were added throughout the cryostat: on the vacuum vessel MLI surface, on the cold mass MLI surface, on the aluminum foil wrapped on the aluminum rib cage, on the magnet support shield boxes, and on parts of the cold mass that were warmest 
during previous thermal cycles.

With all of the improvements described above, a careful thermal analysis of the thermal shield and the thermal intercept system resulted in a predicted cold mass heat load of $2.8 \mathrm{~W}$ at $4.5 \mathrm{~K}$.

Cool-down for the third cold run was started on February 18, 2014. Fig. 4 shows typical cool-down curves of the maximum coil temperature, the minimum coil temperature, and the helium supply temperature. There were no temperature sensors on the coil itself, so the minimum and maximum temperatures were calculated from measured coil resistances with a $10 \mathrm{~mA}$ trickle current. During cool-down with a $0.010 \mathrm{~kg} / \mathrm{s}$ helium flow, the helium supply temperature was continuously regulated to maintain a constant $45 \mathrm{~K}$ below the maximum coil temperature. A $50 \mathrm{~K}$ helium supply temperature was reached after 37 hours of continuous cooling. The $50 \mathrm{~K}$ helium supply temperature was held until the coil temperatures were below $60 \mathrm{~K}$, at which point cool-down was completed with $0.003 \mathrm{~kg} / \mathrm{s}$ of liquid helium. Once liquid helium reached the return end of the magnet, an additional day was required for the cold mass to reach a stable temperature. An additional two days beyond that was required for the MLI and the custom thermal shield to reach thermal equilibrium.

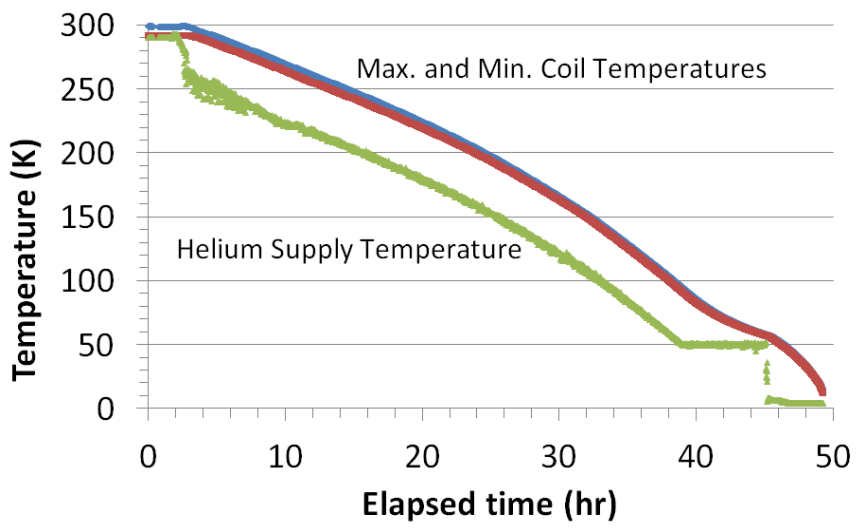

Fig. 4. Typical temperatures as a function of time during a controlled cooldown of the cold mass.

At thermal equilibrium, the insulating vacuum reached $5.3 \times 10^{-4} \mathrm{~Pa}\left(4 \times 10^{-6}\right.$ Torr $)$. The measured MLI surface temperatures were $240-250 \mathrm{~K}$, resulting in a calculated radiation heat flux of approximately $0.5 \mathrm{~W} / \mathrm{m}^{2}$. The temperature of the shield boxes was below $20 \mathrm{~K}$, and the temperature of the aluminum foil on the rib cage was below $10 \mathrm{~K}$. The cold mass reached temperatures of 4.4-5.7 K with the warmest location where one of the current leads entered the cold mass. A temperature map of the cold mass in the horizontal orientation as tested is shown in Fig. 5. Possible reasons for this warm spot are insufficient cooling from the cooling tube that was modified nearby to eliminate a helium to vacuum leak, uneven cooling due to a lateral tilt of the magnet, and a loose cold mass thermal intercept that was found at the conclusion of the test.

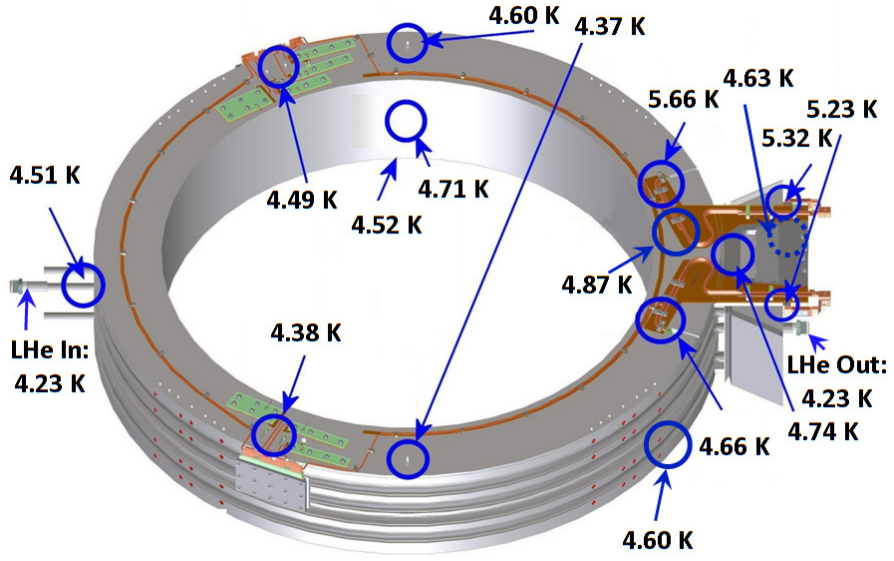

Fig. 5. Temperature map of the cold mass under stable thermal conditions. The maximum temperature was $5.66 \mathrm{~K}$, located where one of the current leads entered the cold mass.

During ramping to $195 \mathrm{~A}$, the current lead intercepts were thermally stable with the warm intercept temperatures increasing from an initial $31 \mathrm{~K}$ to a final $33 \mathrm{~K}$. The cold intercept temperatures increased from an initial $7 \mathrm{~K}$ to a final $8 \mathrm{~K}$.

The total heat load to the cold mass was calculated by stopping the liquid helium flow and measuring the warm-up rate. The results are shown in Fig. 6. The calculated heat load was steady at $1.8 \mathrm{~W}$ for $30 \mathrm{~min}$ before it began rising as the thermal shield started to warm.

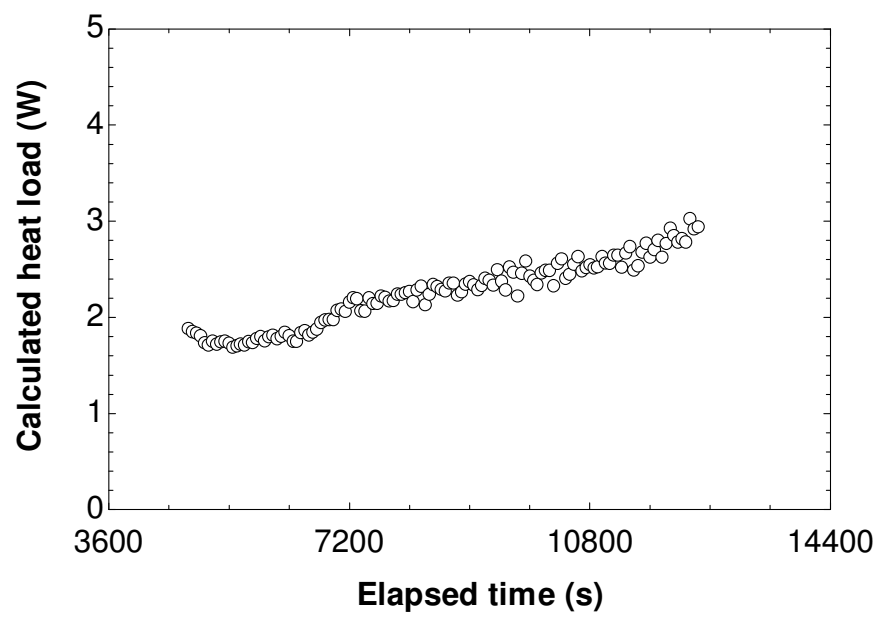

Fig. 6. Calculated cold mass heat load as a function of time. The heat load increases over time as the thermal shield surrounding the cold mass begins to warm and becomes less effective.

\section{Mechanical Performance}

The mechanical behavior of the coupling coil magnet was monitored during cool-down and excitation using strain gauge type SK-13-060PB-35. A minimum set of 4 gauges, including temperature compensators, were placed around the inner bobbin and 4 around the outer structure shell to measure hoop strain. Placed approximately 90 degrees apart the gauges on the outer shell were numbered 1, 3, 5, 7 and correspondingly 2, 4, 6, 8 on the inner bobbin as shown in Fig. 7. All gauges 
were mounted after the coil was already wound and therefore do not reference a free mechanical state. An initial cold compressive reference stress in the bobbin of $-80 \mathrm{MPa}$ was assumed from ANSYS calculations based on $70 \mathrm{MPa}$ of tension used during winding. The outer structural shell was placed over the coil in a loose fashion and any gaps were filled with epoxy.

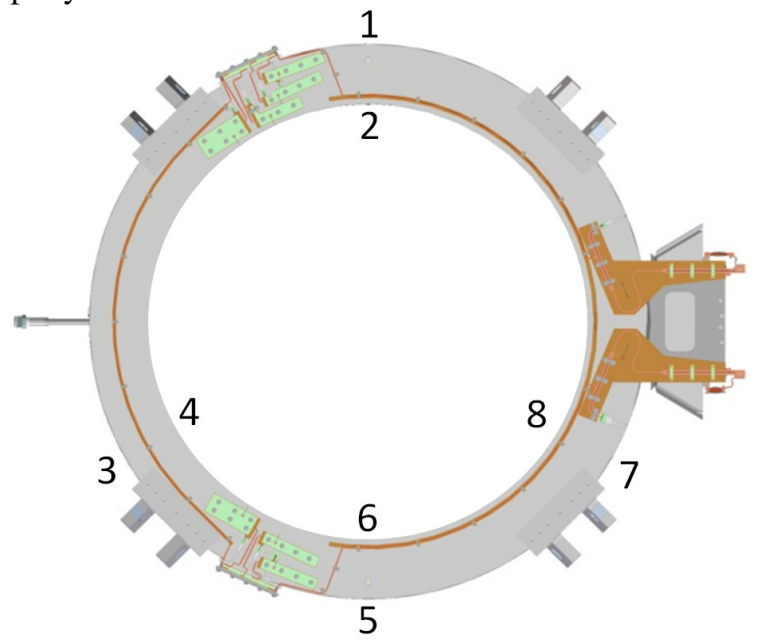

Fig. 7. Strain gauge positions on the cold mass.

Stress on the outer shell during the first 5 training quenches is plotted in Fig. 8. The stress with respect to the applied current squared is expected to behave in a linear fashion. However, during the first ramp the quench Q1 behavior indicated a coil settling effect against the outer shell and a ratcheting effect. The following quenches exhibited a much more linear behavior. The gauges on the inner bobbin behaved somewhat differently during excitation with some gauges indicating a departure from linearity. The departure from linearity occurred at certain currents where Lorentz forces exceeded local mechanical restraining forces, and the coil was forced to tear away from its structure. These behaviors have been seen before in other magnet tests [6] and are attributed to insufficient pre-compression. The coil separation and detachment from the inner structure is usually a source of training. It is also obvious from Fig. 9 that the detachment was not symmetric around the coil and some gauges such as SG6 continued to behave linearly as expected.

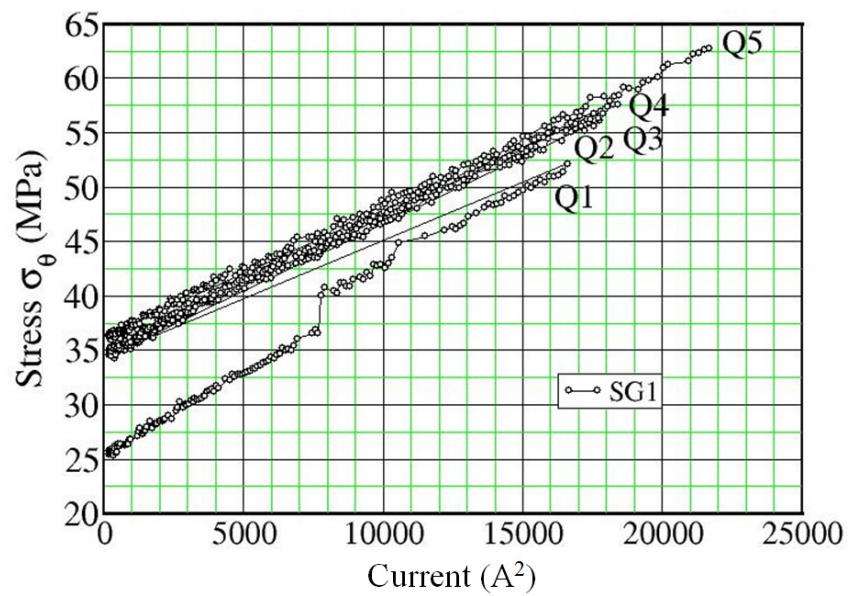

Fig. 8. Outer shell stress at the SG1 position as a function of current squared during the first 5 training quenches. There is an initial settling during the first ramp to quench Q1.

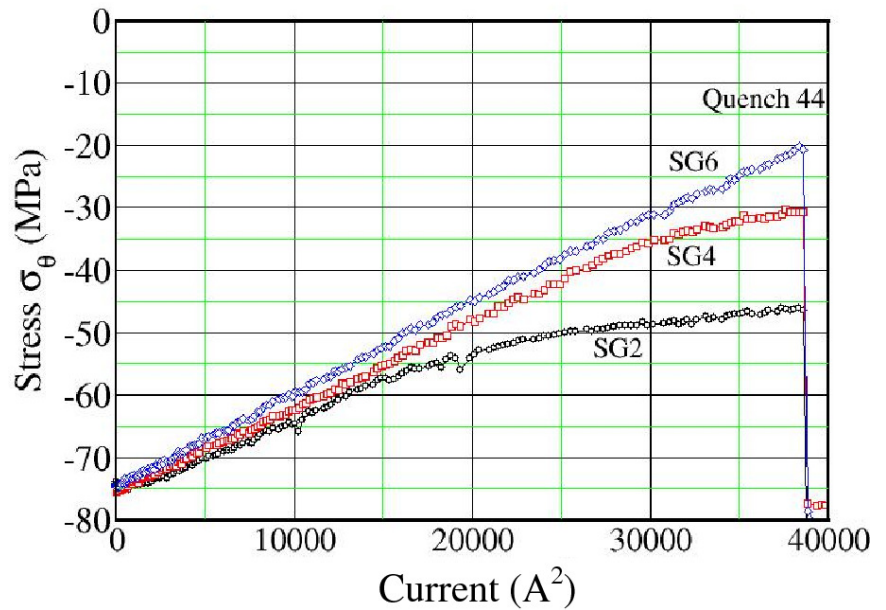

Fig. 9. Outer shell stress at the SG2, SG4, and SG6 positions as a function of current squared during the quench Q44 ramp. The different stress rates indicate that the coil did not separate uniformly from the inner structure.

\section{FUTURE WORK}

The Solenoid Test Facility is presently being modified in preparation for testing a prototype Production Solenoid (PS) [7] for the Fermilab $\mu 2 \mathrm{e}$ project. These modifications include integration of high-temperature superconductor power leads. This will be followed by testing of a $\mu 2 \mathrm{e}$ Transport Solenoid (TS) prototype and the TS production modules, by which time a permanent thermal shield will be installed in the cryostat.

\section{CONCLUSION}

The thermal performance of the Fermilab Solenoid Test Facility while testing the first MICE coupling coil with a custom thermal shield exceeded expectations with a cold mass heat load of only $1.8 \mathrm{~W}$. However, performance was ultimately limited by local cooling conditions at one of the magnet current leads.

The training behavior as shown in Fig. 10 and described in [8] suggests the coil was not firmly held in the structure, a typical conclusion when training is excessive, as it was here. The strain gauge data confirms that, at least partially, insufficient pre-stress was responsible for the coil lifting and separating from the bobbin. This conclusion is also supported by the many training quenches starting in the first coil section attached to the bobbin inner diameter.

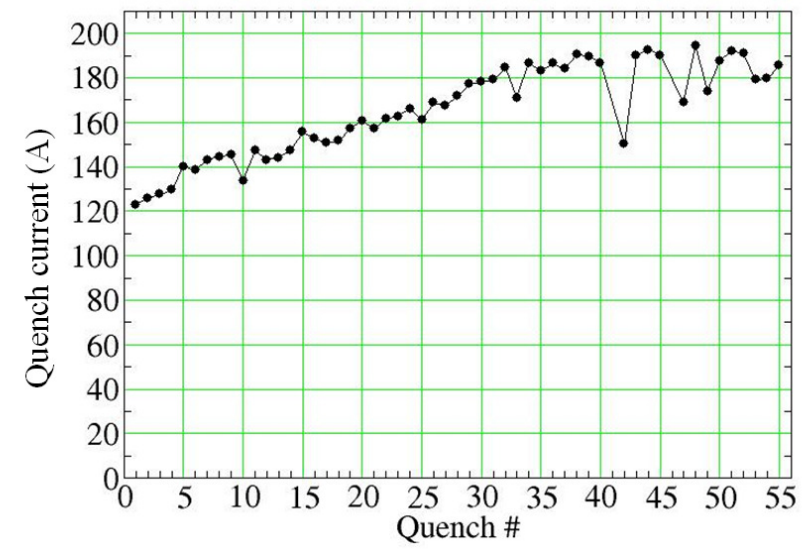

Fig. 10. Quench training of the MICE coupling coil solenoid. 


\section{REFERENCES}

[1] L. Wang et al., "Design and Construction of a Prototype Solenoid Coil for MICE Coupling Magnets," IEEE Transaction on Applied Superconductivity, Vol. 20, no. 3, pp. 373-376, June 2010.

[2] L. Wang et al., "Design and Construction of Test Coils for MICE Coupling Solenoid Magnet," IEEE Transaction on Applied Superconductivity, Vol. 19, no. 3, pp. 1340-1343, June 2009.

[3] L. Wang et al., "Progress on the MuCOOL and MICE Coupling Coils," Proc. PAC 2009 (Vancouver, BC, Canada, May 2009), pp. 289-291.

[4] M. A. Green et al., "Progress on the design of the coupling coils for MICE and MUCOOL," Proc. PAC 2007 (Albuquerque, NM, USA, June 2007), pp. 500-502.

[5] R. Rabehl et al., "A Cryogenic Test Stand for Large Superconducting Solenoid Magnets," Adv. in Cryogenic Engineering, vol. 59A, pp. 215222, 2014.

[6] H. Felice et al., "Performance of a Nb3Sn Quadrupole Under High Stress," IEEE Transaction on Applied Superconductivity, Vol. 21, no. 3, pp. 1849-1853, June 2011.

[7] M. Yoshida et al., "Development of a Radiation Resistant Superconducting Solenoid Magnet for mu-e Conversion Experiments," IEEE Trans. Appl. Supercond. vol. 23, no. 3, Jun. 2013, Art. No. 4101404.

[8] M. Tartaglia, et al., "Electrical and Quench Performance of the First MICE Coupling Coil," IEEE Trans. Appl. Supercond. submitted for publication. 